\title{
CAPTURA DE LULAS (MOLLUSCA: CEPHALOPODA) PELA PESCA INDUSTRIAL DESEMBARCADA EM SANTOS: COMPARAÇÃO APÓS 4 DÉCADAS
}

\author{
GASALLA, M.A.; POSTUMA, F.A. \& TOMÁS, A.R.G. \\ Instituto de Pesca (APTA/SAA/SP). Av. Bartolomeu de Gusmão, 192. Ponta da Praia, 11030-906 \\ Santos, SP. E-mail: mgasalla@osite.com.br
}

\begin{abstract}
Gasalla, M.A.; Postuma, F.A. \& Tomás, A.R.G. 2005. Squid catches (Mollusca: Cephalopoda) by industrial fishing landed in Santos: comparison after 4 decades. Braz. J. Aquat. Sci. Technol. 9(2):5-8. ISSN 1808-7035. Analysis of the temporal variation of fishery resources' landed catch is essential to monitor changes and alterations in the fisheries and related aquatic ecosystems. Thus, analyzing catches after 4 decades represents a comparison between an initial industrial fishing off Southeastern Brazil and a present exploitation phase, considered as decline, and is particularly uncommon due to the atypical data availability. Through the analysis of 1959 and 1999 Santos landing data, this study aims to identify: (a) fleets and gears where squids occurred, with related proportions, (b) differences in the squid's fishery between the two periods, and (c) squids fishing grounds used by the industrial fleets in both periods. Squids were caught by pink-shrimp bottom-trawlers, but also occurred in the sea-bob shrimp fleet, pair-bottom trawlers and purse-seiners, especially in 1959. Differences between both periods can be clearly seen. In 1959, squid fishing areas were more restricted and in lower depths, and in 1999, the areas were broader and deeper. The decrease of fishing boats, and the significant increase of squids catch volume can be verified, where its relative abundance (kg/tow) was 400 times greater in 1999, showing a great availability increase of those resources in the later period or differences on exploitation technology between periods. The existence of both "bottom-up" control mechanism (oceanographic variation-recruitment-fishery production) within the fishery ecosystem, and "top-down" control (fishermen-fisheriesstructure of marine communities) should be taken into account in the interpretation on the squids abundance variation.
\end{abstract}

Keywords: Squids, Cephalopods, Fisheries, Fishery resources, Landings

\section{INTRODUÇÃO}

Variações temporais da captura de recursos pesqueiros estão relacionadas à(s): (1) disponibilidade dos mesmos e a outros fatores oceanográficos, (2) respostas dos estoques pesqueiros às remoções geradas pela pesca, (3) alteração na estrutura das comunidades derivadas de ações antrópicas, e (4) mudanças nas decisões dos pescadores, como escolha dos portos de desembarque e evolução do interesse comercial. Sua análise é fundamental para monitorar mudanças ou alterações nas pescarias e nos ecossistemas marinhos.

A pesca de cefalópodes loliginídeos (lulas) ocorre em ecossistemas costeiros e de plataforma continental, e representa importância crescente na costa Sudeste do Brasil, onde concentrações significativas de Loligo plei e Loligo sanpaulensis representam recursos pesqueiros costeiros potenciais (Costa \& Haimovici, 1990, Pérez, 1999, Gasalla \& Marques, 2000).

Artesanalmente, as lulas são capturadas no verão, junto a ilhas costeiras, através de linha-de-mão com zangarilhos, em pequenas embarcações. De forma industrial, estes recursos ocorrem como fauna acompanhante ou captura acessória da frota pesqueira, principalmente a de arrasto-de-fundo.
Analisar a situação das capturas após 4 décadas de pesca, representa uma comparação entre a fase ainda inicial da pesca industrial no Sudeste do Brasil, e a fase atual, que pode ser considerada como de declínio, e é bastante incomum devido à rara disponibilidade de dados pretéritos.

Este estudo visa, através da análise de dados disponíveis da pesca de 1959 e 1999, identificar: (a) em quais frotas ou artes de pesca ocorreram, de fato, lulas, e em que proporção, (b) diferenças na pesca de lulas entre os dois períodos, e (c) os locais de pesca utilizados pela frota industrial onde foram capturadas lulas nos dois períodos.

\section{MATERIAL E MÉTODOS}

As informações foram obtidas através de consulta ao acervo de dados estatísticos de desembarque do Instituto de Pesca, em Santos, SP. Os dados de 1959 e 1999 foram digitalizados e checados individualmente por frota pesqueira. A reconstrução do local de captura para geo-referenciamento, foi realizada a partir das informações (local, profundidade e distância da costa) fornecidas pelos mestres das embarcações na ocasião da entrevista local rotineira realizada historica- 
mente pelos coletores de dados estatísticos do Instituto de Pesca.

Os dados foram digitalizados e analisados através da padronização no cálculo de captura (em kg), esforço (número de lances onde o recurso lula ocorreu) e cpue (captura por unidade de esforço).

As espécies incluídas na categoria computada nos desembarques como "lulas" são Loligo plei e Loligo sanpaulensis. Lolliguncula brevis, embora possa ocorrer eventualmente, não atinge volume de desembarque.

\section{RESULTADOS E DISCUSSÃO}

A grande diferença entre a captura de lulas em 1959 e 1999, assim como a respectiva participação das frotas pesqueiras correspondentes, podem ser visualizadas na Figura 1. Nos dois períodos, exemplares de lulas ocorreram na pesca das seguintes frotas: parelhas (cujo alvo são os peixes demersais), traineiras (especialmente para sardinhas), arrasteiros de camarão sete-barbas e especialmente na frota de arrasteiros de camarão-rosa.

A distribuição percentual da captura de lulas por frota pesqueira é melhor representada na Figura 2. Verifica-se que nos dois períodos, a maior parte da captura de lulas refere-se à frota de camarão-rosa (70 e 97 $\%$, respectivamente nos dois períodos). Em 1959, porém, a participação dos barcos de parelha teve maior destaque do que em 1999 (28 e 3\%, respectivamente nos dois períodos). As frotas de camarão sete-barbas e de traineiras, tiveram também maior participação na captura de lulas em 1959, do que em 1999, ainda que pequena em relação às demais.

Foi capturado um total de $3802 \mathrm{~kg}$ de lulas nos barcos que desembarcaram na região de Santos em 1959. Já em 1999, a quantidade superou as 400 toneladas. Com relação ao esforço pesqueiro correspondente, em número de barcos atuantes e número de lances, verificou-se que os dados analisados correspondem, em 1959, a um total de 492 embarcações de pesca, e em 1999, a 239 barcos. O número de lances total realizado pelos 4 tipos de frota também variou de um total de 11946 lances, em 1959, para 27885 lances, em 1999. Assim sendo, podem ser observados os valores da captura total por unidade de esforço total (CPUE), em número de lances (Tabela 1).

Ao analisar o desempenho da frota total, a cpue em 1999 excedeu à de 1959 em 400 vezes, o que poderia sugerir uma maior disponibilidade destes organismos em 1999. No entanto, dados de cpue devem ser utilizados com ressalva para exprimir abundância relativa, em casos onde possam ter ocorrido mudanças tecnológicas na frota, e conseqüentemente condições diferentes de capturabilidade. Sabe-se, por exem-
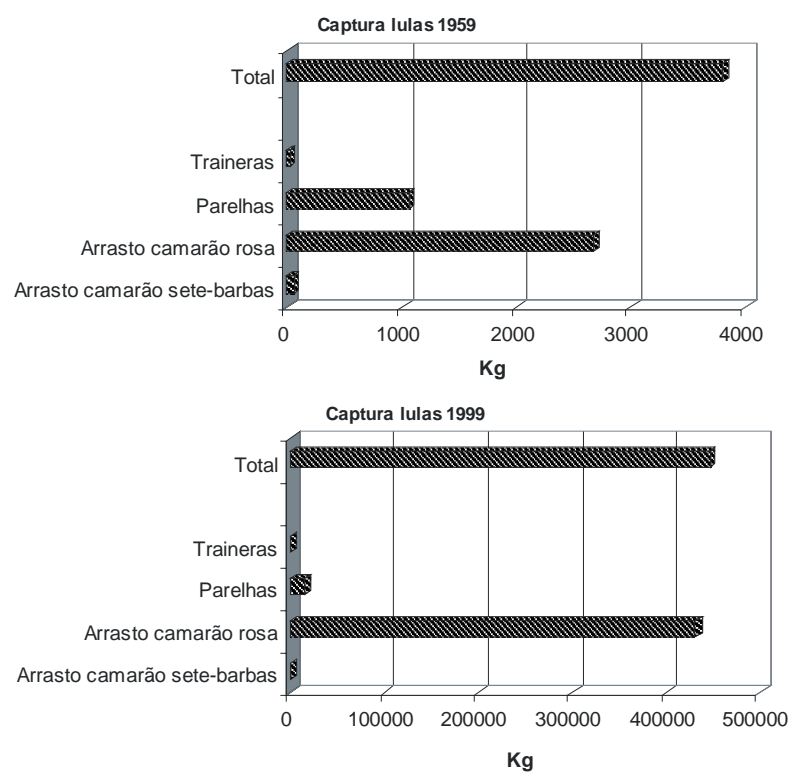

Figura 1 - Captura de lulas (em kg) por frota desembarcada em Santos em 1959 e 1999.
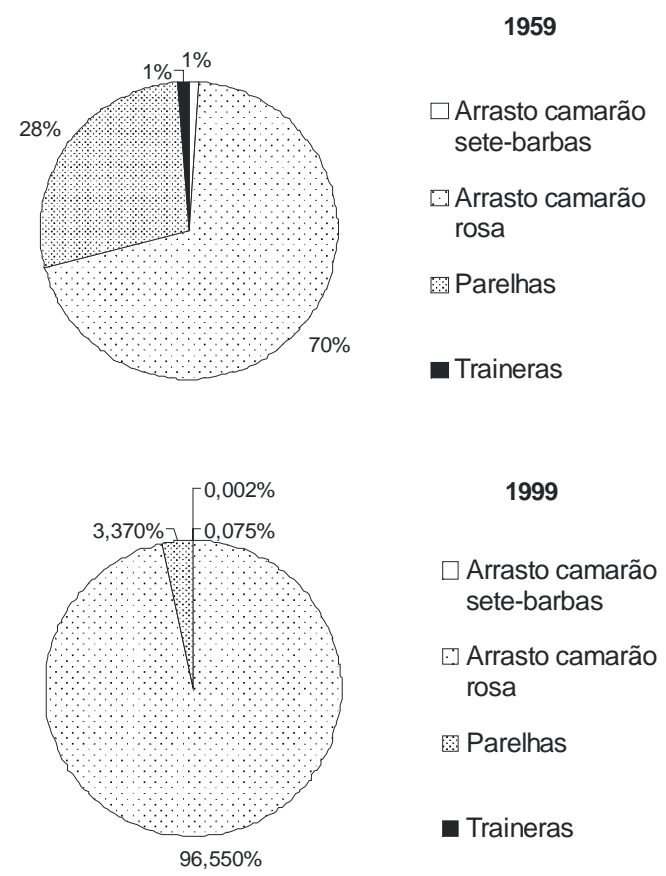

Figura 2 - Importância percentual da participação das frotas pesqueiras na captura de lulas, desembarcadas na região de Santos em 1959 e 1999.

plo, que em 1959, não existiam ainda arrasteiros duplos, e é provável que algumas condições tecnológicas da pesca tenham sido inferiores nessa época.

Cabe comentar, também, que não existem informações disponíveis sobre rejeito de lulas a bordo das embarcações. Sabe-se, contudo, que hoje em dia as 
lulas são recursos alternativos interessantes, raramente rejeitados. Relatórios da pesca da década de 1959 indicam que as lulas eram muito apreciadas, pois encontravam mercado rápido, logo após o desembarque, o que nos faz acreditar que o rejeito a bordo não deveria ser também comum neste período, mesmo que não sendo consideradas recursos-alvo.

Quanto aos locais de pesca onde ocorreram lulas, os mapas apresentados na Figura 3, ilustram os pontos de coleta por frota. Verifica-se que as lulas são capturadas em baixas profundidades (ver isóbata de $100 \mathrm{~m}$ ), na faixa onde atua a frota industrial costeira. $O$ maior número de locais de pesca em 1999 poderia ser explicado pelo maior detalhamento dos dados estatísticos recentes, que em 1959 apenas eram representados em blocos de pesca de maior amplitude. No entanto, sabe-se que o esforço de pesca, a área de procura, assim como a área varrida tende a ser maior em 1999, tanto pela maior escassez dos recursos pesqueiros como pelo provável melhor desempenho das embarcações. De modo geral, a captura de lulas acompanha o deslocamento normal das frotas industriais, aparecendo como fauna acompanhante das espécies-alvo. Observa-se que em 1999, praticamente todas as frotas atuaram em maiores profundidades do que em 1959. Isto era esperado, tanto pelo maior poder de pesca dos barcos como pelo próprio estresse ambiental da zona costeira. A frota de camarão-rosa capturou lulas em toda a sua área de atuação, desde o Rio de Janeiro até Santa Catarina. Já as traineiras, enquanto em 1959 capturaram lulas dentro da Baía da Ilha Grande, em 1999, cujo porte dos barcos atuais é maior, foram pescadas na região externa próxima a Cabo Frio. Porém, pode-se verificar que em 1959, as capturas das pareIhas tiveram maior amplitude de deslocamento, pois a frota de Santos capturava, naquele período, peixes demersais principalmente na região ao Sul. Já em 1999, as capturas de lulas com parelhas, restringiram-se à região norte da plataforma Sudeste (Fig. 3).

Embora a frota de parelhas demonstrasse no passado maior participação na captura de lulas, os índices mais recentes e maiores de abundância de lulas, nessa frota, acenam à possibilidade de uma maior disponibilidade destes cefalópodes no ambiente utilizado pela frota de arrasto demersal (Tabela 1 ).

As maiores capturas de lulas por unidade de esforço, de todas as frotas industriais de 1999 (Tabela 1), poderiam indicar também uma maior disponibilidade desses organismos nesse ano. Contudo, essa variação na abundância pode ser apenas considerada como hipótese, devido à limitação existente da análise de cpues. De fato, outras análises, como a aplicação de

Tabela 1 - Captura por unidade de esforço média de lulas, em kg/lance de pesca, para cada uma das frotas em que ocorreram lulas, nos dois períodos.

\begin{tabular}{lcc}
\hline Frota pesqueira industrial & CPUE lulas 1959 & CPUE lulas 1999 \\
\hline Arrasto de camarão sete barbas & 0,10 & 0,650 \\
Arrasto de camarão-rosa & 0,58 & 17,60 \\
\hline Arrasto de parelhas & 1,29 & 10,84 \\
\hline Traineiras & 0,33 & 0,004 \\
\hline Total & 0,04 & 16,02
\end{tabular}
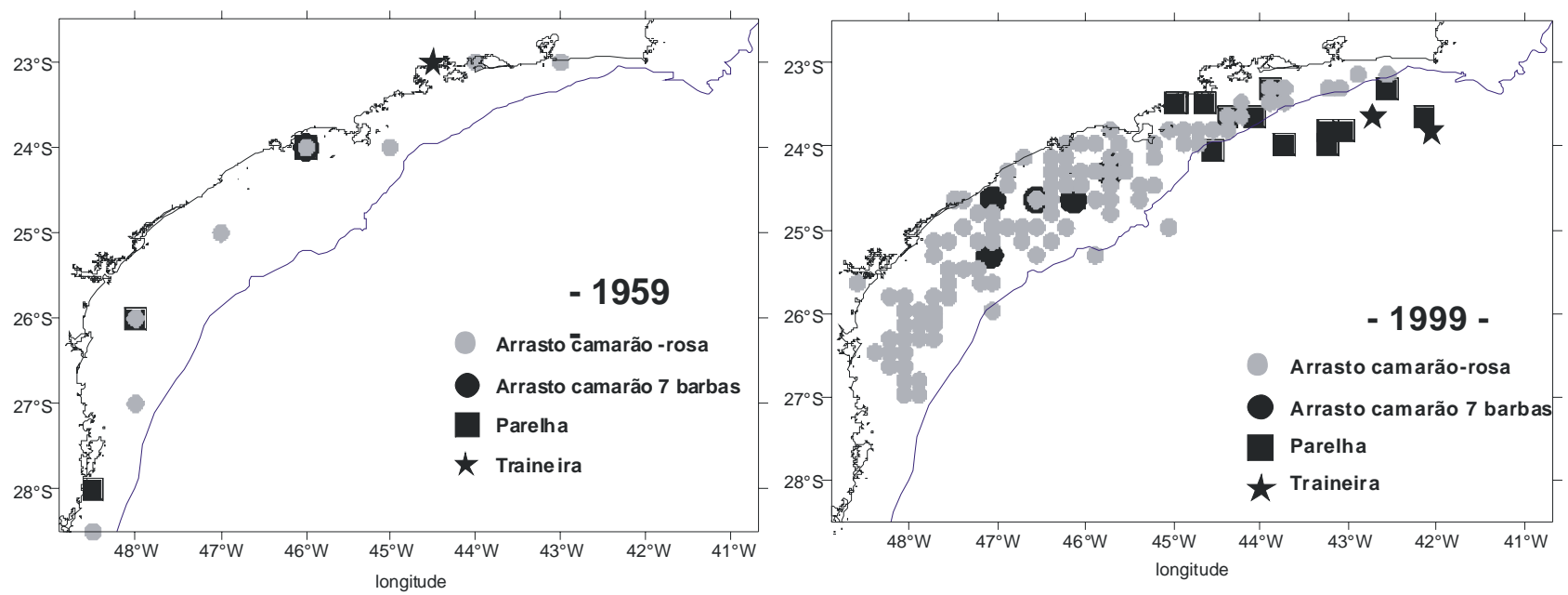

Figura 3 - Locais de pesca onde ocorreram lulas, por frota pesqueira, em 1959 e 1999. 
métodos de padronização de esforços, como os modelos lineares generalizados (i.e. Pérez, 2002), seriam mais indicadas para refletir as mudanças na abundância relativa, mas que foram, aqui, inviáveis pelo tipo de dados disponíveis.

Nesta interpretação, e pese a ausência de uma padronização do esforço, de qualquer maneira, o aumento no volume de desembarque de lulas deve estar bastante relacionado com a pesada pesca dos arrasteiros (Perez \& Pezzuto, 1998), representando uma situação semelhante a outras regiões consideradas sobre-exploradas pela pesca (Caddy \& Rodhouse, 1998; Christensen, 1998). Por outro lado, um possível aumento na abundância de lulas, poderia estar relacionado, inclusive também, com oscilações oceanográficas como El Niño (Tomás et al., 2000), ou com variações naturais habituais em cefalópodos (Caddy, 1983).

Nesse sentido, diversos fatores atuam no ecossistema pesqueiro, que podem explicar as variações nas capturas e nas CPUEs dos recursos pesqueiros. A existência de mecanismos internos de controle tanto "bottom-up" (fatores oceanográficos-recrutamento-produção pesqueira) como "top-down" (pescadores-pesca-estrutura das comunidades marinhas) devem ser sempre levados em conta nas interpretações (Gasalla, 2004; Gasalla \& Rossi-Wongtschowski, 2004).

\section{CONCLUSÕES}

A captura de lulas pela frota industrial que desembarcou em Santos tanto em 1959 como 1999, deuse principalmente pelos barcos arrasteiros de camarão-rosa, mas também ocorreu na frota do camarão setebarbas, dos arrasteiros de parelhas e das traineiras. $A$ área de pesca estendeu-se desde a Baia da llha Grande até o Cabo de Santa Marta, em baixas profundidades, em 1959, e desde o Sul do Cabo Frio até o Norte de Santa Catarina, em profundidades maiores em 1999. As diferenças da pesca entre os dois períodos são claramente observadas. Além das capturas maiores em 1999, levanta-se a hipótese de uma possível maior disponibilidade desse recurso na área de atuação da frota de arrasto de fundo, que não pôde ser devidamente comprovada devido à limitação dos dados existentes.

\section{AGRADECIMENTOS}

Às equipes do Laboratório de Estatística do Instituto de Pesca, pela importante tarefa de arquivar historicamente as informações sobre a pesca desembarcada no Estado de São Paulo. À International Foundation for Science, IFS, pelo apoio financeiro ao projeto de pesquisa em que este estudo está inserido.

\section{REFERÊNCIAS}

Caddy, J.F. 1983. The cephalopods: factors relevant to their population dynamics and to the assessment and management. FAO Fish.Tec.Pap. 231: 454 p.

Caddy J.F. \& Rodhouse, P.G. 1998. Cephalopod and Groundfish Landings: Evidence for Ecological Change in Global Fisheries? Rev.Fish.Biol.Fish. 8(4): 431-444.

Christensen, V. 1998. Fishery-induced changes in a marine ecosytem: insight from models of the Gulf of Thailand. J. Fish Biol. 53(suppl A): 128-142.

Costa, P. A. \& Haimovici, M. 1990. A pesca de polvos e lulas no litoral do Rio de Janeiro. Ciência e Cultura 42 (12): 1124-1130.

Gasalla M.A. 2004. Impactos da pesca industrial no ecossistema da plataforma continental interna do Sudeste do Brasil: a abordagem ecossistêmica e a integração do conhecimento. Tese de doutorado, Instituto Oceanográfico, Universidade de São Paulo, $275 \mathrm{p}$.

Gasalla, M.A. \& Marques, C.A. 2000. Contribuição ao estudo do ciclo reprodutivo da lula Loligo plei (Cephalopoda: Loliginidae) no Sudeste do Brasil. IIDeterminação da época de reprodução. Anais da XII Semana Nacional de Oceanografia, Itajaí. p. 673675.

Gasalla M.A. \& Rossi-Wongtschowski, C.L.D.B. 2004. Contribution of ecosystem analysis to investigating the effects of changes in fishing strategies in the South Brazil Bight coastal ecosystem. Ecological Modelling 172 (2-4): 283-306.

Pérez, J.A.A. 1999. Padronização do esforço da pesca de arrasto em Santa Catarina: O caso da pesca da lula, Loligo plei. Notas técnicas Facimar 3: 47-56.

Perez J.A.A. 2002. Biomass dynamics of the squid Loligo plei and the development of a small-scale seasonal fishery off southern Brazil. Bull.Mar.Sci. 71(2): 633-651.

Perez J.A.A. \& Pezzuto P.R. 1998. Valuable shellfish species in the by-catch of shrimp fishery in southern Brazil: spatial and temporal patterns. J.Shellfish Res. 17(1): 303-309.

Tomás, R.G.; Carneiro, M.H. \& Gasalla, M.A. 2000. Cephalopods in the fishery of the Southeastern Brazil $X$ Environment conditions during last decade: are there relationships to ENSO phenomena? CIAC 2000, Cephalopods Biomass and Production, Aberdeen, UK. 